\title{
Low vitamin D levels and non-alcoholic fatty liver disease, evidence for their independent association in men in East China: a cross-sectional study (Survey on Prevalence in East China for Metabolic Diseases and Risk Factors (SPECT-China))
}

\author{
Hua-Ling Zhai†, Ning-Jian Wang†, Bing Han, Qin Li, Yi Chen, Chun-Fang Zhu, Ying-Chao Chen, \\ Fang-Zhen Xia, Zhen Cang, Chao-Xia Zhu, Meng Lu and Ying-Li Lu* \\ Institute and Department of Endocrinology and Metabolism, Shanghai Ninth People's Hospital, Shanghai Jiao Tong University \\ School of Medicine, Shanghai 200011, People's Republic of China
}

(Submitted 13 September 2015 - Final revision received 4 January 2016 - Accepted 19 January 2016 - First published online 18 February 2016)

\section{Abstract}

Recent studies have suggested an association between vitamin D and non-alcoholic fatty liver disease (NAFLD); however, some results are subject to debate. This study was carried out to evaluate the correlation between NAFLD and vitamin D in men and women in East China. The data were obtained from a cross-sectional study that focused on the health and metabolic status of adults in sixteen areas of East China. According to ultrasonic assessments, the patients were divided into normal and NAFLD groups. Demographic characteristics and biochemical measurements were obtained. Binary logistic regression analysis was used to explore the association. In total, 5066 subjects were enrolled, and 2193 (43.3\%) were diagnosed with NAFLD; 84.56\% of the subjects showed vitamin D deficiency. Subjects with high vitamin D levels had a lower prevalence of NAFLD, particularly male subjects. Within the highest quartile of vitamin D levels, the prevalence of NAFLD was $40 \cdot 8 \%$, whereas the lowest quartile of vitamin D levels showed a prevalence of $62.2 \%$, which was unchanged in women across the vitamin D levels. Binary logistic analysis showed that decreased vitamin D levels were associated with an increased risk of NAFLD (OR 1.54; 95\% CI 1.26, 1.88). This study suggests that vitamin D levels are significantly associated with NAFLD and that vitamin D acts as an independent factor for NAFLD prevalence, particularly in males in East China. Vitamin D interventional treatment might be a new target for controlling NAFLD; elucidating the mechanism requires further research.

\section{Key words: Vitamin D: Non-alcoholic fatty liver disease: BMI: Homoeostasis model assessment: Insulin resistance}

Non-alcoholic fatty liver disease (NAFLD), alternatively known as metabolic fatty liver disease, is associated with insulin resistance, dyslipidaemia, obesity and the metabolic syndrome $^{(1)}$. In parallel with the obesity epidemic, NAFLD has become one of the most common causes of chronic liver disease in the world. Up to $30 \%$ of adults are affected by NAFLD in America ${ }^{(2)}$. A study from China reported that the prevalence of NAFLD was as high as $20 \%{ }^{(3)}$. Vitamin D deficiency (VDD) can be a result of inadequate or limited exposure to sunlight, improper diet or problems related to absorption of vitamin D. Certain diseases can impair vitamin D conversion into its active form. Some of these conditions include kidney disease, liver disease or some hereditary diseases. VDD usually results in bad mineralisation of bones, leading to bone softening, osteomalacia, rickets and osteoporosis. As VDD is a risk factor for developing these metabolic deficiencies and has been linked to disorders in bone metabolism, more recent studies have suggested that obesity and visceral obesity occur with VDD. The potential association between VDD and NAFLD has been examined in recent studies. Mohamed et al. ${ }^{(4)}$ reported that VDD is an important risk factor for NAFLD, and Kong et al. ${ }^{(5)}$ observed that VDD might up-regulate endogenous fatty acid synthesis in non-alcoholic steatohepatitis (NASH) through impaired enterohepatic circulation and that administration of 1,25-dihydroxyvitamin $\mathrm{D}_{3}$ corrected the NASH phenotypes in line with suppression of hepatic lipogenesis and inflammation. Rhee et al. ${ }^{(6)}$ reported that participants with higher serum 25-hydroxyvitamin $\mathrm{D}_{3}$ $\left(25(\mathrm{OH}) \mathrm{D}_{3}\right)$ showed a significantly reduced risk for NAFLD compared with low $25(\mathrm{OH}) \mathrm{D}_{3}$ groups, independent of obesity and the metabolic syndrome. Sharifi et $a l .{ }^{(7)}$ and Targher et al. ${ }^{(8)}$ suggest that treatment of vitamin $\mathrm{D}_{3}$ deficiency may prevent or treat NAFLD. Bril et al. ${ }^{(9)}$ observed the plasma vitamin D levels of 239 patients who were divided into three groups; however, no positive association between vitamin D and NAFLD was observed. The inconsistency of these studies might arise from

Abbreviations: 25(OH)D $\mathrm{D}_{3}$, 25-hydroxyvitamin $\mathrm{D}_{3}$; HbA1c, glycated $\mathrm{Hb}$; HOMA-IR, homoeostatic model assessment for insulin resistance; NAFLD non-alcoholic fatty liver disease; US, ultrasonography; VDD, vitamin D deficiency.

* Corresponding author: Y.-L. Lu, fax +86 216313 6856, email luyingli2008@126.com

$\dagger$ Both authors contributed equally to this work. 
differences in the study populations, sample sizes and methods for diagnosing NAFLD.

We carried out a large-sample, cross-sectional survey to analyse the association between vitamin D levels and NAFLD in East China.

\section{Methods}

Survey on Prevalence in East China for Metabolic Diseases and Risk Factors-China study

East China comprises Shanghai and seven provinces (sixteen areas) with a population of approximately 395 million, accounting for $29.2 \%$ of the people in China. People of Han Chinese ethnicity comprise $99.5 \%$ of the population. Survey on Prevalence in East China for Metabolic Diseases and Risk Factors-China is a population-based, cross-sectional survey of the prevalence of metabolic diseases and risk factors in East China. The registration number of the trial is ChiCTR-ECS-14005052 (www.chictr.org). We collection was performed by the same staff group from the Department of Endocrinology of the Shanghai Ninth People's Hospital, which is affiliated to the Shanghai Jiao Tong University School of Medicine. The staff members were trained according to a standard protocol that familiarised them with the specific tools and methods used in the survey. The trained staff members used a questionnaire to collect information on demographic characteristics, medical history and lifestyle risk factors. From February to June 2014, this study was performed in an urban area in Shanghai and Jiangxi Province and in a rural area in Shanghai, Zhejiang and Jiangxi Province ${ }^{(10)}$. Adults aged 18 years and above who were Chinese citizens and had lived in their current residence for 6 months or longer were selected and invited to join our study. Individuals with severe communication problems, acute illness and unwillingness to participate were excluded from the study in the first exclusion phase. A total of 7200 participants remained after the first phase of exclusion. In the next step, we excluded participants who had missing laboratory results ( $n$ 183) and questionnaire data ( $n$ 112) and were younger than 18 years of age ( $n$ 6). Subsequently, 6899 subjects were enrolled. Further, subjects with history of excessive alcohol consumption and self-reported viral hepatitis were excluded. In addition, patients taking vitamin supplements and without vitamin D results were excluded. After these patients were excluded, 5066 patients were included into the final cohort of this study (Fig. 1 shows the protocol of the study design). The study protocol was approved by the Ethics Committee of the Shanghai Ninth People's Hospital, Shanghai Jiao Tong University School of Medicine (approval number 2013(86)). The research was conducted according to the Declaration of Helsinki. All the participants provided their written informed consent before data collection.

\section{Measurements}

Body weight, height and blood pressure were measured using standard methods, as previously described ${ }^{(11)}$. Waist:hip ratio was calculated from the waist circumference measured on bare skin as the narrowest circumference between the 10th rib and

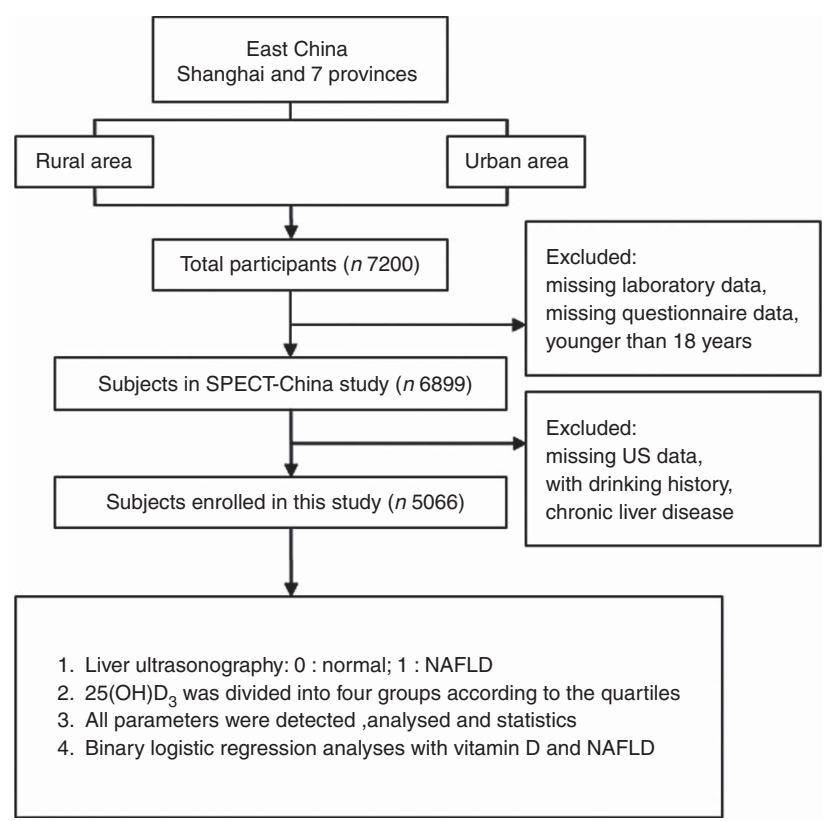

Fig. 1. Flow chart of sampling frame and participants selected from Survey on Prevalence in East China for Metabolic Diseases and Risk Factors (SPECT)China. Flow chart of SPECT-China. In total, with several exclusion criteria, 5066 participants were enrolled. NAFLD, non-alcoholic fatty liver disease; $25(\mathrm{OH}) \mathrm{D}_{3}, 25$-hydroxyvitamin $\mathrm{D}_{3}$.

the iliac crest and hip circumference at the widest point of the gluteal muscles. BMI was calculated as weight in kilograms divided by height in metres squared.

\section{Serum sample collection and biochemical analysis}

Venous blood samples were drawn after an overnight fast of at least $8 \mathrm{~h}$. The blood samples for the plasma glucose test were collected into vacuum tubes with anticoagulant sodium fluoride and centrifuged immediately, within $1 \mathrm{~h}$ after collection. The blood samples were stored at $-20^{\circ} \mathrm{C}$ after collection and shipped within $2-4 \mathrm{~h}$ of collection by air on dry ice to a central laboratory, which was certified by the College of American Pathologists. The glycated $\mathrm{Hb}$ (HbA1c) level was assessed by HPLC (MQ-2000PT; Shanghai HuiZhong Medical Technology Co. Ltd.). The plasma glucose and lipid profile including total cholesterol, TAG, HDLcholesterol and LDL-cholesterol were measured using a Beckman Coulter AU 680 (Beckman Coulter) instrument. A chemiluminescence immunoassay (Siemens ADVIA Centaur XP; Siemens) was used to detect $25(\mathrm{OH}) \mathrm{D}_{3}$. Insulin resistance was estimated by calculating the homoeostatic model assessment for insulin resistance (HOMA-IR) index ${ }^{(12)}$, and the fasting insulin (FINS, pmol/ 1) $\times$ fasting glucose $(\mathrm{mg} / \mathrm{dl}) /(22.5 \times 6.965)$ levels were measured.

\section{Non-alcoholic fatty liver disease evaluation}

Liver ultrasonography (US) was performed to assess the degree of steatosis. The US examinations were performed by the same three operators by consensus. All imaging studies were performed in the fundamental brightness mode (B-mode). US findings were analysed based on criteria $^{(13)}$ for US diagnosis in humans, according to the changes in liver echogenicity, 
classified into two patterns: normal, homogeneous liver parenchyma with medium-level echogenicity and a regular hepatic surface; and fatty liver, discrete coarse and heterogeneous parenchymal echogenicity and dotted, irregular or nodular hepatic liver surface.

\section{Statistics}

Statistical Package for the Social Sciences version 19.0 software was used for the analysis. All the analyses were two-sided; $P<0.05$ was considered to be statistically significant. All reported $P$ values were two-sided. The general characteristics are summarised as means and standard deviations for continuous variables or as a number with proportion for categorical variables. To test for differences in characteristics between the participants with and without NAFLD, and among 25(OH)D quantiles, the Kruskal-Wallis test and ANOVA were used for continuous variables with skewed distribution and normal distribution, respectively.

OR and $95 \% \mathrm{CI}$ were calculated using logistic regression to determine the risk of NAFLD for each quartile of $25(\mathrm{OH}) \mathrm{D}$. Model 1 controlled for age (continuous variable) and sex. Model 2 additionally controlled for smoking status, BMI, alanine aminotransferase (ALT) level, systolic blood pressure (SBP), TAG, HDL-cholesterol, LDL-cholesterol and diabetes.

\section{Results}

\section{General characteristics of the study subjects}

NAFLD was found in 2193 subjects (43.3\%). In the NAFLD group, the number of participants undergoing antihypertensive and hypolipemic treatment were 292 (13.32\%) and 18 (0.82\%), whereas in non-NAFLD group the numbers were $159(5.53 \%)$ and $7(0.24 \%)$, respectively. The subjects with NAFLD showed slightly lower vitamin D levels (40.05 (SD 9.83) $v$.
$40 \cdot 98$ (sD 10.80) nmol/l) and more unfavourable metabolic profiles. The NAFLD group had higher HbA1c (5.48 (SD 0.95) $v .5 .20$ (SD 0.70$) \%$ ) and elevated fasting plasma glucose (FPG) levels (5.83 (sD 1.50) v. 5.42 (SD 1.04) mmol/l). In addition, the NAFLD group showed significantly higher BMI (25.96 (SD 3.38) $v$. $22.62(\mathrm{SD} 2.95) \mathrm{kg} / \mathrm{m}^{2}$ ) and HOMA-IR levels (1.98 (SD 2.68) $v$. 1.26 (SD 1.30)) than did the non-NAFLD group. Further characteristics of the study subjects with and without NAFLD are presented in Table 1.

\section{The prevalence of different vitamin D status in the normal and non-alcoholic fatty liver disease groups}

The average of $25(\mathrm{OH}) \mathrm{D}_{3}$ levels in our study was $40 \cdot 58 \mathrm{nmol} / 1$, which did not reach adequate $25(\mathrm{OH}) \mathrm{D}_{3}$ levels $(50 \mathrm{nmol} / \mathrm{l})$, and $84.56 \%$ of the subjects showed a $25(\mathrm{OH}) \mathrm{D}_{3}$ level $<50 \mathrm{nmol} / \mathrm{l}$, which indicated vitamin $\mathrm{D}$ insufficiency; the percentage of vitamin $\mathrm{D}$ insufficiency in the normal group and the NAFLD group was similar $(82.91 v .86 .73 \%)$. Fig. 2 shows that VDD in men is not as severe as that in women (78.05 v. 87.55\%); however, men with NAFLD showed a lower than normal vitamin $\mathrm{D}$ level $(84.87 v .70 \cdot 05 \%)$, and this finding was not observed in women (88.02 v. 87.35\%).

\section{Correlations between vitamin $D$ and the prevalence of non-alcoholic fatty liver disease}

To more effectively reveal the relationship between vitamin D and NAFLD, we analysed the association between serum vitamin D levels and the prevalence of NAFLD in men and women (Fig. 3). We divided the subjects into four groups according to their vitamin D level quartiles. We observed a significantly higher prevalence of NAFLD in men with lower vitamin $\mathrm{D}(63.1 v \cdot 38 \cdot 8 \%)$, whereas the association in women was not obvious (38.9 v. 37.8\%).

Table 1. Demographic and general characteristics of the study participants*

(Mean values and standard deviations for continuous variables)

\begin{tabular}{|c|c|c|c|c|c|c|c|}
\hline \multirow[b]{2}{*}{ Variables } & \multicolumn{2}{|c|}{ Total } & \multicolumn{2}{|c|}{ Non-NAFLD } & \multicolumn{2}{|c|}{ NAFLD } & \multirow[b]{2}{*}{$P$} \\
\hline & Mean & SD & Mean & SD & Mean & SD & \\
\hline$n$ & \multicolumn{2}{|c|}{5066} & \multicolumn{2}{|c|}{2873} & \multicolumn{2}{|c|}{2193} & \\
\hline Age (years) & $52 \cdot 15$ & 13.95 & $50 \cdot 66$ & 14.57 & $54 \cdot 10$ & $12 \cdot 85$ & $<0.001$ \\
\hline $\mathrm{BMI}\left(\mathrm{kg} / \mathrm{m}^{2}\right)$ & 24.07 & 3.55 & 22.62 & 2.95 & $25 \cdot 96$ & 3.38 & $<0.001$ \\
\hline WHR & 0.85 & 0.20 & 0.83 & 0.16 & 0.88 & 0.23 & $<0.001$ \\
\hline $\mathrm{FPG}(\mathrm{mmol} / \mathrm{l})$ & $5 \cdot 60$ & 1.28 & 5.42 & 1.04 & 5.83 & 1.50 & $<0.001$ \\
\hline $\mathrm{HbA1c}(\%)$ & 5.32 & 0.83 & $5 \cdot 20$ & 0.70 & 5.48 & 0.95 & $<0.001$ \\
\hline $\mathrm{TC}(\mathrm{mmol} / \mathrm{l})$ & 5.05 & 1.01 & 4.93 & 0.95 & 5.19 & 1.07 & $<0.001$ \\
\hline TAG $(\mathrm{mmol} / \mathrm{l})$ & 1.57 & 1.45 & 1.28 & 0.83 & 1.95 & 1.93 & $<0.001$ \\
\hline HDL-cholesterol ( $\mathrm{mmol} / \mathrm{l})$ & 1.46 & 0.32 & 1.52 & 0.31 & 1.37 & 0.31 & $<0.001$ \\
\hline LDL-cholesterol (mmol/l) & 2.92 & 0.72 & $2 \cdot 81$ & 0.70 & 3.05 & 0.73 & $<0.001$ \\
\hline FINS $(p m o l / l)$ & 41.88 & $42 \cdot 16$ & $35 \cdot 36$ & $29 \cdot 17$ & $50 \cdot 42$ & 53.50 & $<0.001$ \\
\hline HOMA-IR & 1.57 & 2.05 & 1.26 & 1.30 & 1.98 & 2.68 & $<0.001$ \\
\hline ALT (IU/I) & 21.52 & $15 \cdot 22$ & $18 \cdot 86$ & $12 \cdot 86$ & 25.02 & $17 \cdot 24$ & $<0.001$ \\
\hline AST (IU/I) & 24.49 & 9.93 & 23.65 & 8.90 & $25 \cdot 60$ & 11.05 & $<0.001$ \\
\hline $25(\mathrm{OH}) \mathrm{D}(\mathrm{nmol} / \mathrm{l})$ & $40 \cdot 58$ & $10 \cdot 40$ & $40 \cdot 98$ & $10 \cdot 80$ & 40.05 & 9.83 & $<0.01$ \\
\hline
\end{tabular}

NAFLD, non-alcoholic fatty liver disease; WHR, waist:hip ratio; FPG, fasting plasma glucose; HbA1c, glycated Hb; TC, total cholesterol; FINS, fasting insulin; HOMA-IR, homoeostasis model assessment-insulin resistance; ALT, alanine aminotransferase; AST, aspartate transaminase; $25(\mathrm{OH}) \mathrm{D}, 25-\mathrm{hydroxyvitamin} \mathrm{D.}$

* The Mann-Whitney $U$ and Student's $t$ test was used for skewed and normal distribution, respectively. 

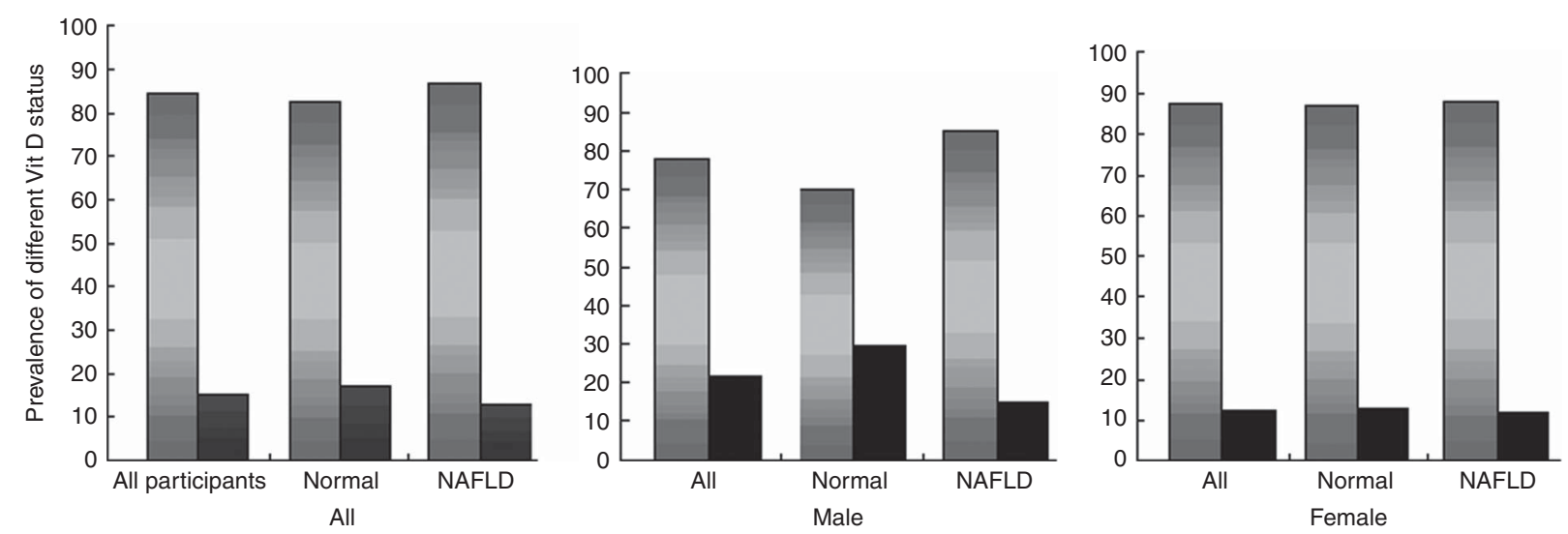

Fig. 2. Prevalence of vitamin $D(V i t D)$ insufficiency in different participants. Prevalence of serum Vit $D$ insufficiency among total participants, the normal group and the non-alcoholic fatty liver disease (NAFLD) group. These values were also diagraph analysed in male and female groups. Vit $D$ insufficiency was defined as levels $<50 \mathrm{nmol} / \mathrm{l}(\square)$, and Vit D sufficiency was defined as levels $>50 \mathrm{nmol} / \mathrm{l}(\square)$.

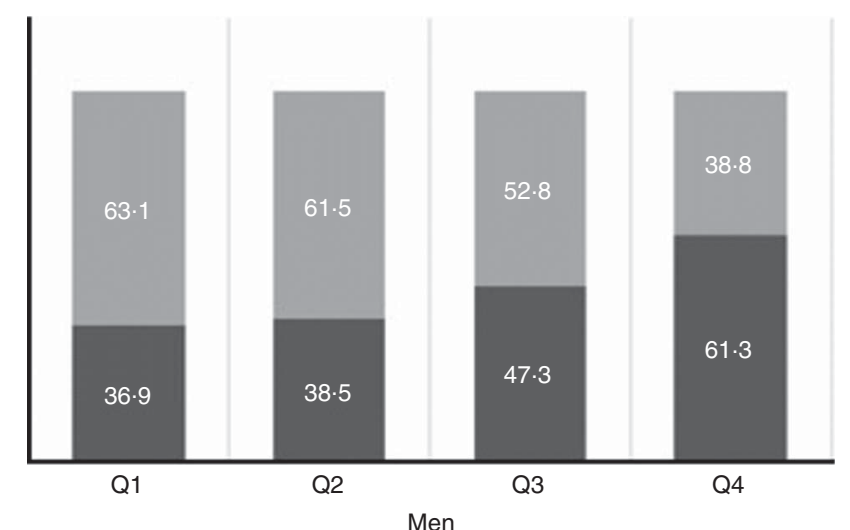

improve. In women, the BMI and FPG showed no significance among the different vitamin D groups, and only the TAG level was ameliorated with ascending vitamin D levels.

\section{The association between vitamin $D$ and non-alcoholic fatty} liver disease by logistic regression analyses

Table 3 shows the association between vitamin D and NAFLD according to our binary logistic regression analyses. In the multivariate-adjusted model 1 , after adjustment for age and sex, the OR for NAFLD in the lowest compared with the highest quartile of serum vitamin D was 1.64 (95\% CI 1.39, 1.94). After further adjustment for the smoking status, BMI, ALT, SBP, TAG, HDL-cholesterol, LDL-cholesterol and diabetes, the risk for the prevalence of NAFLD decreased across the vitamin D quartiles, and the OR in the lowest compared with the highest quartile of serum vitamin D was 1.54 (95\% CI 1.26, 1.76). As the OR were attenuated, the gradually increasing trend with decreasing vitamin D showed significance.

\section{Discussion}

In our study, we found that the NAFLD prevalence was $43.3 \%$ in East China, which exceeded the prevalence that had previously been reported in China ${ }^{(14)}$. The subjects with NAFLD showed slightly lower vitamin D levels and more unfavourable metabolic profiles including a higher HbA1c level as well as elevated FPG, BMI and HOMA-IR. The average $25(\mathrm{OH}) \mathrm{D}_{3}$ level in our study was $40.58 \mathrm{nmol} / \mathrm{l}$, which did not reach the adequate $25(\mathrm{OH}) \mathrm{D}_{3}$ levels $(50 \mathrm{nmol} / \mathrm{l}): 2 \cdot 13 \%$ of the subjects showed a $25(\mathrm{OH}) \mathrm{D}_{3}$ level $<25 \mathrm{nmol} / 1$, which indicated VDD, $82.43 \%$ had a level $<50 \mathrm{nmol} / 1$ and $1.05 \%$ demonstrated a level above the $75 \mathrm{nmol} / 1$ target suggested by vitamin D experts ${ }^{(15,16)}$. FINS in the NAFLD group was apparently increased compared with the NAFLD group, which may be explained by the mechanism of the metabolic syndrome with insulin resistance. VDD and NAFLD have indirect and direct associations with obesity and a sedentary lifestyle, and therefore it is expected that VDD would co-exist with NAFLD. Some studies have explored 
Table 2. The characteristics of the study sample by 25 -hydroxyvitamin $D(25(\mathrm{OH}) \mathrm{D})$ quartiles $(\mathrm{Q})$ in men and women*

(Mean values and standard deviations for continuous variables)

\begin{tabular}{|c|c|c|c|c|c|c|c|c|c|}
\hline & \multicolumn{8}{|c|}{ Vitamin D level (mU/l) } & \multirow[b]{3}{*}{$P$} \\
\hline & \multicolumn{2}{|c|}{ Q1 ( $\leq 35.09)$} & \multicolumn{2}{|c|}{ Q2 (35.10-41.35) } & \multicolumn{2}{|c|}{ Q3 (41.36-48.77) } & \multicolumn{2}{|c|}{ Q4 ( $\geq 48.78)$} & \\
\hline & Mean & SD & Mean & SD & Mean & SD & Mean & SD & \\
\hline \multicolumn{10}{|l|}{ Men } \\
\hline$n$ & \multicolumn{2}{|c|}{401} & \multicolumn{2}{|c|}{401} & \multicolumn{2}{|c|}{401} & \multicolumn{2}{|c|}{400} & \\
\hline Age (years) & 49.06 & $15 \cdot 44$ & $49 \cdot 74$ & 13.69 & $52 \cdot 82$ & $13 \cdot 81$ & 58.03 & 14.56 & $<0.001$ \\
\hline $\mathrm{BMI}\left(\mathrm{kg} / \mathrm{m}^{2}\right)$ & 24.29 & 3.42 & $24 \cdot 80$ & 3.55 & 24.49 & $3 \cdot 22$ & 23.98 & 3.26 & $<0.01$ \\
\hline WHR & 0.88 & 0.08 & 0.88 & 0.08 & 0.92 & 0.64 & 0.89 & 0.08 & 0.19 \\
\hline $\mathrm{FPG}(\mathrm{mmol} / \mathrm{l})$ & $5 \cdot 62$ & 1.55 & 5.62 & 1.49 & 5.61 & 1.30 & 5.60 & 1.21 & 0.99 \\
\hline HbA1c (\%) & 5.45 & 1.07 & $5 \cdot 38$ & 1.04 & $5 \cdot 37$ & 0.88 & 5.41 & 0.78 & 0.57 \\
\hline TAG $(\mathrm{mmol} / \mathrm{l})$ & $2 \cdot 14$ & 2.88 & 1.87 & 1.32 & 1.61 & $1 \cdot 16$ & 1.41 & 1.03 & $<0.001$ \\
\hline HDL-cholesterol (mmol/l) & 1.31 & 0.30 & 1.29 & 0.27 & 1.32 & 0.28 & 1.36 & 0.30 & $<0.01$ \\
\hline LDL-cholesterol (mmol/l) & 2.95 & 0.76 & 3.00 & 0.69 & $2 \cdot 86$ & 0.65 & $2 \cdot 80$ & 0.64 & $<0.001$ \\
\hline FINS (pmol/l) & $42 \cdot 67$ & 48.44 & $44 \cdot 25$ & 44.74 & 38.66 & 32.43 & 38.02 & 56.71 & $<0.001$ \\
\hline HOMA-IR & 1.62 & 2.41 & 1.66 & 2.05 & 1.45 & 1.53 & 1.44 & 2.43 & $<0.01$ \\
\hline ALT (IU/I) & $28 \cdot 47$ & $22 \cdot 04$ & $27 \cdot 98$ & $19 \cdot 70$ & $26 \cdot 13$ & $15 \cdot 48$ & 23.28 & 15.52 & $<0.001$ \\
\hline \multirow[t]{2}{*}{ AST (IU/I) } & $26 \cdot 26$ & $10 \cdot 19$ & $26 \cdot 25$ & $12 \cdot 85$ & $26 \cdot 40$ & $11 \cdot 35$ & $26 \cdot 37$ & 9.63 & 0.40 \\
\hline & \multicolumn{2}{|c|}{ Q1 $(\leq 32.69)$} & \multicolumn{2}{|c|}{ Q2 (32.70-37.95) } & \multicolumn{2}{|c|}{ Q3 (37.96-44.31) } & \multicolumn{2}{|c|}{ Q4 ( $\geq 44 \cdot 32)$} & $P$ \\
\hline \multicolumn{10}{|l|}{ Women } \\
\hline$n$ & \multicolumn{2}{|c|}{867} & \multicolumn{2}{|c|}{866} & \multicolumn{2}{|c|}{866} & \multicolumn{2}{|c|}{864} & \\
\hline Age (years) & 51.07 & 14.02 & $50 \cdot 74$ & $12 \cdot 73$ & 51.06 & 13.02 & 55.25 & 13.84 & $<0.001$ \\
\hline BMI $\left(\mathrm{kg} / \mathrm{m}^{2}\right)$ & 23.75 & 3.70 & 23.85 & 3.57 & 24.01 & 3.71 & 24.06 & 3.48 & 0.27 \\
\hline WHR & 0.82 & 0.07 & 0.82 & 0.07 & 0.82 & 0.08 & 0.85 & 0.08 & $<0.001$ \\
\hline $\mathrm{FPG}(\mathrm{mmol} / \mathrm{l})$ & $5 \cdot 64$ & 1.20 & $5 \cdot 60$ & 1.31 & $5 \cdot 55$ & $1 \cdot 18$ & 5.57 & $1 \cdot 19$ & 0.54 \\
\hline $\mathrm{HbA1c}(\%)$ & $5 \cdot 21$ & 0.85 & $5 \cdot 27$ & 0.73 & 5.29 & 0.74 & 5.39 & 0.75 & $<0.001$ \\
\hline TAG $(\mathrm{mmol} / \mathrm{l})$ & 1.61 & 1.44 & 1.53 & 1.61 & 1.40 & 0.89 & 1.38 & 0.93 & $<0.001$ \\
\hline HDL-cholesterol (mmol/l) & 1.51 & 0.32 & 1.52 & 0.31 & 1.52 & 0.31 & 1.53 & 0.32 & 0.69 \\
\hline LDL-cholesterol (mmol/l) & $2 \cdot 85$ & 0.72 & 2.92 & 0.78 & 2.94 & 0.69 & 2.97 & 0.74 & $<0.01$ \\
\hline FINS (pmol/l) & 44.35 & 36.84 & $40 \cdot 31$ & $26 \cdot 40$ & 43.55 & 55.43 & $41 \cdot 14$ & 35.78 & 0.12 \\
\hline HOMA-IR & 1.68 & 2.03 & 1.50 & 1.38 & 1.65 & $2 \cdot 79$ & 1.50 & 1.53 & 0.09 \\
\hline ALT (IU/I) & 19.50 & $12 \cdot 35$ & $19 \cdot 36$ & 14.79 & $19 \cdot 17$ & 11.57 & 18.91 & $12 \cdot 36$ & 0.88 \\
\hline AST (IU/I) & 23.86 & $10 \cdot 20$ & 23.45 & 9.65 & $23 \cdot 60$ & 8.85 & $23 \cdot 70$ & 8.15 & 0.26 \\
\hline
\end{tabular}

WHR, waist:hip ratio; FPG, fasting plasma glucose; HbA1c, glycated Hb; FINS, fasting insulin; HOMA-IR, homoeostasis model assessment-insulin resistance; ALT, alanine aminotransferase; AST, aspartate transaminase.

* The Kruskal-Wallis test and ANOVA were used for continuous variables with skewed distribution and normal distribution, respectively. 
the potentially causative relationship between VDD and NAFLD, and clinical trials have identified trends in the epidemiology of VDD and NAFLD. In a study of 262 patients referred to an endocrinology clinic, the relationship between NAFLD and VDD was confirmed, regardless of age, sex, TAG levels and insulin resistance ${ }^{(17)}$. Targher et al. ${ }^{(18)}$ verified this point and confirmed the relationship between liver histology and vitamin D. In our study, we found that VDD had an independent relationship (OR 1.54; 95\% CI 1.26, 1.76) with NAFLD. These results are in accordance with the studies of Hao et al. ${ }^{(19)}$ and Lu et al. ${ }^{(20)}$ who concluded that high serum $25(\mathrm{OH}) \mathrm{D}_{3}$ levels were a protective factor against NAFLD after adjusting for risk factors. How does it work, and what is the mechanism? Kong et al. ${ }^{(5)}$ suggested a novel mechanism for NASH development, by which VDD down-regulates ileal apical sodium-dependent bile acid cotransporter (iASBT) expression, resulting in a poor bile acid pool and elevation of hepatic lipogenesis and inflammation. In conclusion, vitamin $\mathrm{D}$ and bile acid sequestration may be explored as new strategies to treat or prevent NASH. The inverse association between serum vitamin D levels and the metabolic syndrome may also be frequently explained by the following. First, the answer might lie in the mechanism of the 'two-hit' hypothesis of the pathogenesis of NAFLD, which includes a number of metabolic pathways. VDD could positively affect these pathways, including hormonal, immunological and cellular differentiation mechanisms. Second, VDD could affect adipocytokines (i.e. adiponectin, resistin and omentin) and pro-inflammatory cytokines (i.e. TNF- $\alpha$ and IL-6), which are secreted by adipose tissue and have important roles in the formation of NAFLD ${ }^{(21-23)}$ Sharifi et al ${ }^{(24)}$ has reported that improved vitamin D status led to amelioration in serum C-reactive protein (CRP) and malondialdehyde in patients with NAFLD, and vitamin D might be considered an adjunct therapy to attenuate systemic inflammation and lipid peroxidation with other treatments of NAFLD patients. We found that higher vitamin D levels exerted a protective effect on NAFLD, and we recommend higher daily intake of vitamin D.

Table 2 shows that BMI increased with the vitamin D quartiles, especially in women, but there was no significance, whereas in men the change was opposite and in accordance with other studies. The reason may be the testosterone levels in males, which may play an important role. Although this is a surprising outcome, it is a fact, explained in the study by Wang et al. ${ }^{(25,26)}$.

Another finding is that VDD in men is not as severe as that in women, but the NAFLD in men displayed an obviously lack of normal vitamin D levels, whereas this difference was not observed in women. We also observed a significantly higher prevalence of NAFLD in subjects with lower vitamin D levels among men, whereas this association in women was not obvious. This suggests that vitamin D supplements may ameliorate the fatty liver prevalence in men better than in women, but the mechanism still requires further exploration.

\section{Limitations}

Our study had some limitations. Although liver biopsy is regarded as the gold standard for detecting hepatic statuses ${ }^{(27)}$, liver biopsy was not feasible in the case of this population-based study. US is the most suitable diagnostic test of choice for NAFLD because it is

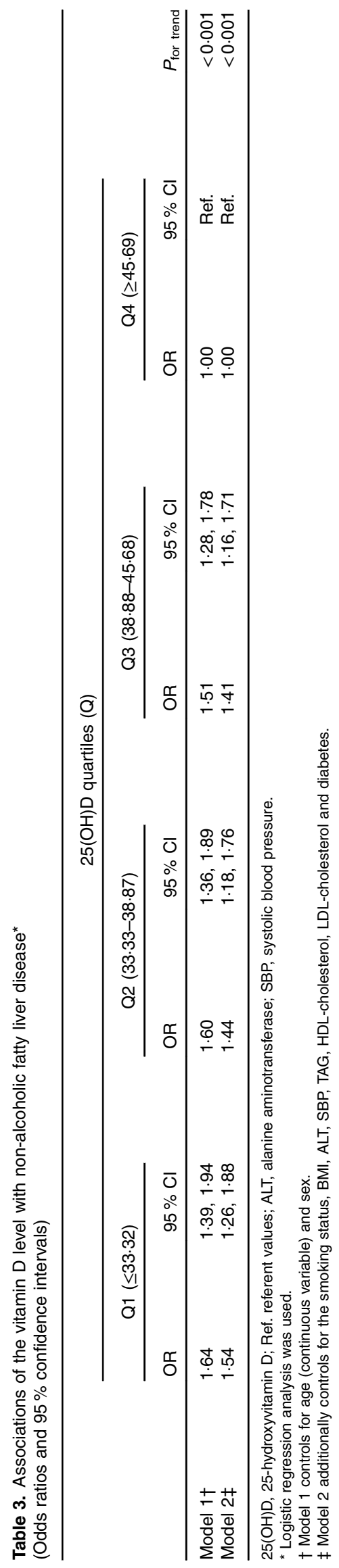


non-invasive, safe, sensitive and specific in terms of identifying fatty infiltration. However, as this was judged by the subjective feeling of the operators, the occurrence may deviate, but our US examinations were performed by the same two operators by consensus so as to minimise the deviation.

In our study, we found that female patients with highest HbA1c and fasting glucose had the highest vitamin D levels, whereas in men the results showed no significance, which is contradictory to previous studies. Zhou \& Ye ${ }^{(28)}$ suggested that low levels of $25(\mathrm{OH}) \mathrm{D}$ is significantly associated with the occurrence of type 2 diabetes mellitus (T2DM) complicated with lower extremity arterial disease. Moreover, Lim et al. ${ }^{(29)}$ also reported that vitamin D metabolism may play a role in T2DM pathogenesis independently of known risk factors. We think this may be related to the age factor in the women group, with increase in age HbA1c levels elevated significantly and diabetes mellitus prevalence increased.

As HOMA index is usually used to calculate insulin resistance in subjects with the metabolic syndrome without diabetes, or in those with diabetes without therapy, the use of HOMA-IR may be debatable in diabetic individuals. However, many studies $^{(30-33)}$ have also demonstrated that HOMA-IR is significantly associated with diabetes.

In our study, 5066 persons were enrolled, among them 539 participants had diabetes, and thirty-eight participants were using insulin treatment. In the sensitivity analyses, we have re-calculated the HOMA-IR among the participants excluding subjects with diabetes, and the results of comparison are in accordance with the former.

\section{Conclusion}

The vitamin D level in most of the population is insufficient, and we have demonstrated the relation between vitamin $\mathrm{D}$ levels and NAFLD. Low vitamin D levels conferred a significantly higher risk of developing NAFLD, especially in men in East China. This association remained significant even after adjusting for the BMI and some risk factors of NAFLD. Thus, it may be worthwhile to monitor serum vitamin D levels to screen for NAFLD, and vitamin D supplementation is recommended. The mechanisms underlying the association between vitamin $\mathrm{D}$ and NAFLD are still largely unclear. Further studies are necessary to uncover new mechanisms linking vitamin D and NAFLD.

\section{Acknowledgements}

The authors thank all the team members and the participants from Shanghai, Zhejiang Province and Jiangxi Province in the Survey on Prevalence in East China for Metabolic Diseases and Risk Factors-China study.

This study was supported, in part, by the National Natural Science Foundation of China (grant numbers 81270885, 81070677 and 81300653); Clinical Potential Subject Construction of Shanghai Jiao Tong University School of Medicine (grant number 2014); the Ministry of Science and Technology in China (grant number 2012CB524906); the Science and Technology Commission of Shanghai Municipality (grant number
14495810700); and funds for outstanding academic leaders in Shanghai (grant number 12XD1403100).

H.-L. Z., N.-J. W., B. H. and Q. L. collected the data and drafted the manuscript. Y. C., C.-F. Z., Y.-C. C., F.-Z. X., Z. C., C.-X. Z. and M. L. participated in the study design and performed the statistical analysis. Y.-L. L. conceived the study and participated in its design and coordination and helped to draft the manuscript. All the authors read and approved the final version of the manuscript.

The authors declare that there are no conflicts of interest.

\section{References}

1. Chalasani N, Younossi Z, Lavine JE, et al. (2012) The diagnosis and management of non-alcoholic fatty liver disease: practice guideline by the American Association for the Study of Liver Diseases, American College of Gastroenterology, and the American Gastroenterological Association. Hepatology 55 , 2005-2023.

2. Williams CD, Stengel J, Asike MI, et al. (2011) Prevalence of nonalcoholic fatty liver disease and nonalcoholic steatohepatitis among a largely middle-aged population utilizing ultrasound and liver biopsy: a prospective study. Gastroenterology 140, 124-131.

3. Wong VW (2013) Nonalcoholic fatty liver disease in Asia: a story of growth. J Gastroenterol Hepatol 28, 18-23.

4. Mohamed AA, Mahmoud S, Ahmed R, et al. (2015) Vitamin D is it a key in Egyptian NAFLD pathogenesis? J Gastroenterol Hepatol 4, 1605-1609.

5. Kong M, Zhu L, Bai L, et al. (2014) Vitamin D deficiency promotes nonalcoholic steatohepatitis through impaired enterohepatic circulation in animal model. Am J Physiol Gastrointest Liver Physiol 307, G883-G893.

6. Rhee EJ, Kim MK, Park SE, et al. (2013) High serum vitamin D levels reduce the risk for nonalcoholic fatty liver disease in healthy men independent of metabolic syndrome. Endocr J 60, 743-752.

7. Sharifi N, Amani R, Hajiani E, et al. (2014) Does vitamin D improve liver enzymes, oxidative stress, and inflammatory biomarkers in adults with non-alcoholic fatty liver disease? A randomized clinical trial. Endocrine 47, 70-80.

8. Targher G, Scorletti E, Mantovani A, et al. (2013) Nonalcoholic fatty liver disease and reduced serum vitamin D(3) levels. Metab Syndr Relat Disord 11, 217-228.

9. Bril F, Maximos M, Portillo-Sanchez P, et al. (2014) Relationship of vitamin $\mathrm{D}$ with insulin resistance and disease severity in non-alcoholic steatohepatitis. J Hepatol 62, 405-411.

10. Wang N, Kuang L, Han B, et al. (2015) Follicle-stimulating hormone associates with prediabetes and diabetes in postmenopausal women. Acta Diabetol (Epublication ahead of print version 12 May 2015).

11. Xu Y, Wang L, He J, et al. (2013) Prevalence and control of diabetes in Chinese adults. JAMA 310, 948-959.

12. Matthews DR, Hosker JP, Rudenski AS, et al. (1985) Homeostasis model assessment: insulin resistance and beta-cell function from fasting plasma glucose and insulin concentration in man. Diabetologia 28, 412-419.

13. Hamaguchi M, Kojima T, Takeda N, et al. (2007) Nonalcoholic fatty liver disease is a novel predictor of cardiovascular disease. World J Gastroenterol 13, 1579-1584.

14. Ong JP \& Younossi ZM (2007) Epidemiology and natural history of NAFLD and NASH. Clin Liver Dis 11, 1-16.

15. Ross AC, Manson JE, Abrams SA, et al. (2011) The 2011 report on dietary reference intakes for calcium and vitamin $\mathrm{d}$ from the institute of medicine: what clinicians need to know. J Clin Endocrinol Metab 96, 53-58. 
16. Holick MF, Binkley NC, Bischoff-Ferrari HA, et al. (2011) Evaluation, treatment, and prevention of vitamin D deficiency: an endocrine society clinical practice guideline. J Clin Endocrinol Metab 96, 1911-1930.

17. Barchetta I, Angelico F, Del Ben M, et al. (2011) Strong association between non alcoholic fatty liver disease (NAFLD) and low 25(OH) vitamin D levels in an adult population with normal serum liver enzymes. BMC Med 9, 85 .

18. Targher G, Bertolini L, Scala L, et al. (2007) Associations between serum 25-hydroxyvitamin $\mathrm{D}_{3}$ concentrations and liver histology in patients with non-alcoholic fatty liver disease. Nutr Metab Cardiovasc Dis 17, 517-524.

19. Hao YP, Ma XJ, Luo YQ, et al. (2014) Serum vitamin D is associated with non-alcoholic fatty liver disease in Chinese males with normal weight and liver enzymes. Acta Pharmacol Sin 35, 1150-1156.

20. Lu Z, Pan X, Hu Y, et al. (2015) Serum vitamin D levels are inversely related with non-alcoholic fatty liver disease independent of visceral obesity in Chinese postmenopausal women. Clin Exp Pharmacol Physiol 42, 139-145.

21. Fantuzzi G (2005) Adipose tissue, adipokines, and inflammation. J Allergy Clin Immunol 115, 911-919.

22. Hotamisligil GS, Shargill NS \& Spiegelman BM (1993) Adipose expression of tumor necrosis factor-alpha: direct role in obesity-linked insulin resistance. Science 259, 87-91.

23. Roth CL, Elfers CT, Figlewicz DP, et al. (2012) Vitamin D deficiency in obese rats exacerbates nonalcoholic fatty liver disease and increases hepatic resistin and toll-like receptor activation. Hepatology 55, 1103-1111.

24. Sharifi N, Amani R, Hajiani E, et al. (2014) Does vitamin D improve liver enzymes, oxidative stress, and inflammatory biomarkers in adults with non-alcoholic fatty liver disease? A randomized clinical trial. Endocrine 47, 70-80.
25. Wang N, Li Q, Han B, et al. (2015) Follicle-stimulating hormone is associated with non-alcoholic fatty liver disease in Chinese women over 55 years old. J Gastroenterol Hepatol (epublication ahead of print version 18 December 2015).

26. Wang N, Han B, Li Q, et al. (2015) Vitamin D is associated with testosterone and hypogonadism in Chinese men: results from a cross-sectional SPECT-China study. Reprod Biol Endocrinol 13, 74

27. Kobyliak N \& Abenavoli L (2014) The role of liver biopsy to assess non-alcoholic fatty liver disease. Rev Recent Clin Trials 9. 159-169.

28. Zhou W \& Ye SD (2015) Relationship between serum 25-hydroxyvitamin D and lower extremity arterial disease in type 2 diabetes mellitus patients and the analysis of the intervention of vitamin D. J Diabetes Res 2015, 815949.

29. Lim S, Kim MJ, Choi SH, et al. (2013) Association of vitamin D deficiency with incidence of type 2 diabetes in high-risk Asian subjects. Am J Clin Nutr 97, 524-530.

30. Mojiminiyi OA \& Abdella NA (2010) Effect of homeostasis model assessment computational method on the definition and associations of insulin resistance. Clin Chem Lab Med 48, 1629-1634.

31. Ghasemi A, Tohidi M, Derakhshan A, et al. (2015) Cut-off points of homeostasis model assessment of insulin resistance, beta-cell function, and fasting serum insulin to identify future type 2 diabetes: Tehran Lipid and Glucose Study. Acta Diabetol 52, 905-915.

32. Morimoto A, Tatsumi Y, Soyano F, et al. (2014) Increase in homeostasis model assessment of insulin resistance (HOMA-IR) had a strong impact on the development of type 2 diabetes in Japanese individuals with impaired insulin secretion: the Saku study. PLOS ONE 9, e105827.

33. Sarafidis PA, Lasaridis AN, Nilsson PM, et al. (2007) Validity and reproducibility of HOMA-IR, 1/HOMA-IR, QUICKI and McAuley's indices in patients with hypertension and type II diabetes. J Hum Hypertens 21, 709-716. 